\title{
Structure of light hypernuclei in the framework of Fermionic Molecular Dynamics
}

\author{
Martin Schaefer ${ }^{\star a b}$, Hans Feldmeier ${ }^{c d}$, Jiri Mares ${ }^{a}$ and Thomas Neff ${ }^{c}$ \\ ${ }^{a}$ Nuclear Physics Institute \\ 25068 Rez, Czech Republic \\ ${ }^{b}$ Czech Technical University in Prague, Faculty of Nuclear Sciences and Physical Engineering \\ Brehova 7, 11519 Prague 1, Czech Republic \\ ${ }^{c}$ GSI Helmholtzzentrum für Schwerionnenforsung GmbH \\ Planckstrasse 1, 64291 Darmstadt, Germany \\ ${ }^{d}$ Frankfurt Institute for Advanced Studies \\ Max-von-Laue-Strasse 1, 60438 Frankfurt, Germany \\ E-mail: m.schafer@ujf.cas.cz
}

\begin{abstract}
We present results of the first variational calculations of the s-shell hypernuclei ${ }_{\Lambda}^{4} \mathrm{He}$ and ${ }_{\Lambda}^{4} \mathrm{H}$ using a recently developed Fermionic Molecular Dynamics (FMD) code. The relevance of the variation of the parity projected trial state $\left(\mathrm{VAP}^{\pi}\right)$ is discussed. We found rather weak dependence of the $\Lambda$ separation energies on the type of the $V_{N N}$ potential. We observed substantial difference between the predicted $\Lambda$ separation energy spectra for various $V_{\Lambda N}$ potentials applied in calculations. The proper choice of $V_{\Lambda N}$ as well as the Fermi momentum $k_{F}$, which enters the YNG $V_{\Lambda N}$ potentials as a parameter, is thus crucial.
\end{abstract}

53rd International Winter Meeting on Nuclear Physics

26-30 January 2015

Bormio, Italy

\footnotetext{
* Speaker.
} 


\section{Introduction}

Hypernuclei are nuclear systems with non-zero strangeness containing besides neutrons and protons also one or more hyperons. Their study provides unique opportunity to test models of baryon-baryon interactions, as well as various nuclear models. Since the $\Lambda$ hyperon is not affected by Pauli blocking in the nucleus, it can penetrate deep in the nuclear interior and thus serves as a probe of the nuclear medium. The $\Lambda N$ scattering data are rather scarce and therefore the form of the $\Lambda N$ potential is not so strictly constrained as in the $N N$ case. Selected $\Lambda$ hypernuclear data are thus used as an input for the $\Lambda N$ interaction models.

There is fair amount of data on $\Lambda$ hypernuclei including their production, structure and decay. The most precise information, including $\gamma$-ray spectra, are available for s-shell and p-shell $\Lambda$ hypernuclei up to mass number $\mathrm{A}=16$ [1].

In this paper we present the first application of the Fermionic Molecular Dynamics model $[2,3]$ in structure calculations of the light s-shell $\Lambda$ hypernuclei ${ }_{\Lambda}^{4} \mathrm{He}$ and ${ }_{\Lambda}^{4} \mathrm{H}$. We studied the effects in the nuclear core caused by the presence of $\Lambda$. We have evaluated the $\Lambda$ separation energy of the hypernuclear ground and excited states for different $N N$ and $\Lambda N$ effective potentials in order to explore model dependence of the calculations.

In Section 2, we briefly introduce the FMD model together with applied projection techniques. Results of hypernuclear structure calculations are discussed in Section 3. We summarize our findings in Section 4 where our future plans are presented as well.

\section{Theoretical approach}

Hypernuclear and nuclear calculations presented in this work are performed within the Fermionic Molecular Dynamics approach. The FMD is a non-relativistic many-body variational model which describes systems of $N$ interacting fermions. The antisymmetrized many-body wave function is adopted in a Slater determinant form

$$
\begin{aligned}
\left\langle x_{1}, \ldots, x_{N} \mid Q\right\rangle & =\frac{1}{N !} \sum_{P_{i}} \operatorname{sgn}\left(P_{i}\right)\left\langle x_{1} \mid q_{P_{i}(1)}\right\rangle \ldots\left\langle x_{N} \mid q_{P_{i}(N)}\right\rangle \\
& =\frac{1}{N !} \operatorname{Det}\left|\begin{array}{ccc}
\left\langle x_{1} \mid q_{1}\right\rangle & \cdots & \left\langle x_{1} \mid q_{N}\right\rangle \\
\vdots & \ddots & \vdots \\
\left\langle x_{N} \mid q_{1}\right\rangle & \cdots & \left\langle x_{N} \mid q_{N}\right\rangle
\end{array}\right|,
\end{aligned}
$$

where $x_{i}$ denotes a generalized coordinate which fully defines space, spin and isospin components of a single-particle state.

The single-particle basis is spanned by Gaussian wave packets

$$
\begin{aligned}
& \left|q_{k}\right\rangle=\left|a_{k}, \vec{b}_{k}\right\rangle \otimes\left|\chi_{k}^{\uparrow}, \chi_{k}^{\downarrow}\right\rangle \otimes\left|\xi_{k}\right\rangle, \\
& \left\langle\vec{x} \mid a_{k}, \vec{b}_{k}\right\rangle=\exp \left(-\frac{\left(\vec{x}-\vec{b}_{k}\right)^{2}}{2 a_{k}}\right),
\end{aligned}
$$

where $\vec{b}_{k}$ stands for position-like complex vector. In contrary to AMD [4] or HyperAMD [5] we use for each state a different complex width parameter $a_{k}$. The spin part $\left|\chi_{k}^{\uparrow}, \chi_{k}^{\downarrow}\right\rangle$ is defined as a most 
general complex spinor to ensure correct rotational properties. The isospin part $\left|\xi_{k}\right\rangle$ remains fixed throughout the entire calculation. Each single particle state is then described by twelve independent variational parameters $q_{k}=\left\{a_{k}, \vec{b}_{k}, \chi_{k}^{\uparrow}, \chi_{k}^{\downarrow}\right\}$.

The Hamiltonian describing a hypernuclear system is defined in a form

$$
\hat{H}=\hat{T}_{N}+\hat{T}_{\Lambda}-\hat{T}_{c m}+\hat{V}_{N N}+\hat{V}_{\Lambda N},
$$

where $\hat{T}_{N}, \hat{T}_{\Lambda}$, and $\hat{T}_{c m}$ denote kinetic energy of nucleons, $\Lambda$, and center of mass motion, respectively. The $\hat{V}_{N N}\left(\hat{V}_{\Lambda N}\right)$ stands for $N N(\Lambda N)$ two-body potential. In this paper we use the effective VolkovV2 [6] $N N$ interaction as well as Malfliet-Tjon V [7] and Afnan-Tang S3M [8] UCOM transformed [9] $\hat{V}_{N N}$ potentials. The hypernuclear part $\hat{V}_{\Lambda N}$ is represented by the G-matrix transformed YNG-NF and YNG-NS Nijmegen two-body $\Lambda N$ potentials [10].

The binding energy and the corresponding state of the nuclear or hypernuclear system are obtained within the time-independent variation method. We minimize the expectation value of the Hamiltonian (2.3) with respect to the parameters $q_{k}$ of each single-particle state $\left|q_{k}\right\rangle$. The binding energy $E_{B}$ of the many-body system is then defined as a minimum

$$
E_{B}=\min _{q_{1}, \ldots, q_{N}} \frac{\langle Q|\hat{H}| Q\rangle}{\langle Q \mid Q\rangle}
$$

under following conditions

$$
<\hat{X}_{c m}>^{2}=0, \quad<\hat{P}_{c m}>^{2}=0, \operatorname{Re}\left(a_{k}\right)>0 .
$$

To solve this problem numerically we have implemented Sequential Quadratic Programming (SQP) method [11].

The FMD state $|Q\rangle$ is in general not an eigenstate of the parity and total angular momentum operators. Hence, we project out a state with particular quantum numbers $J^{\pi}$ by applying on the resulting state of the variation method the parity projector

$$
\hat{P}^{\pi}=\frac{1}{2}(1+\pi \hat{\Pi})
$$

and the total angular momentum projector within the GCM [12]

$$
\hat{P}_{M K}^{J}=\frac{2 J+1}{8 \pi^{2}} \int d \Omega D_{M K}^{J}{ }^{*}(\Omega) \hat{R}(\Omega) .
$$

Given that the parity and total angular momentum projected states $\hat{P}_{M K}^{J} \hat{P}^{\pi}|Q\rangle=\left|Q ; J^{\pi} M K\right\rangle$ are not lineary independent, we obtain orthogonal eigenstates

$$
\left|Q ; J^{\pi} M \kappa\right\rangle=\sum_{K}\left|Q ; J^{\pi} M K\right\rangle C_{K}^{J^{\pi} \kappa}
$$

by the diagonalization of the Hamiltonian (2.3) in a subspace spanned by projected states $\left|Q ; J^{\pi} M K\right\rangle$. This procedure formulates the generalized eigenvalue problem

$$
\begin{gathered}
\sum_{K^{\prime}} H_{K K^{\prime}}^{J \pi} C_{K^{\prime}}^{J^{\pi}}=E^{J^{\pi} K} \sum_{K^{\prime \prime}} N_{K K^{\prime \prime}}^{J^{\pi}} C_{K^{\prime \prime}}^{J^{\pi}}, \\
H_{K, K^{\prime}}^{J^{\pi}}=\left\langle Q ; J^{\pi} M K|\hat{H}| Q ; J^{\pi} M^{\prime} K^{\prime}\right\rangle=\left\langle Q ; \pi\left|\hat{H} \hat{P}_{K K^{\prime}}^{J}\right| Q ; \pi\right\rangle, \\
N_{K, K^{\prime}}^{J^{\pi}}=\left\langle Q ; J^{\pi} M K \mid Q ; J^{\pi} M^{\prime} K^{\prime}\right\rangle=\left\langle Q ; \pi\left|\hat{P}_{K K^{\prime}}^{J}\right| Q ; \pi\right\rangle .
\end{gathered}
$$


As a result we obtain coefficients $C^{J^{\pi} \kappa}$ defining eigenstates (2.8) and corresponding binding energies $E^{J^{\pi} \kappa}$.

The minimization procedure could be further improved by applying the projection techniques [13] to the trial FMD state already before the variation, as will be discussed in the next section.

\section{Results}

In this contribution, we present results of the first calculations of the s-shell hypernuclei ${ }_{\Lambda}^{4} \mathrm{He}$ and ${ }_{\Lambda}^{4} \mathrm{H}$ within the recently developed FMD code. Calculated $\Lambda$ separation energies $B_{\Lambda}$ are compared with the experimental values of $B_{\Lambda}$ in the $0^{+}\left(1^{+}\right)$state, $B_{\Lambda} \exp .\left({ }_{\Lambda}^{4} \mathrm{He} ; 0^{+}\right)=2.39 \pm$ $0.03(1.15 \pm 0.04) \mathrm{MeV}$ and $B_{\Lambda}$ exp. $\left({ }_{\Lambda}^{4} \mathrm{H} ; 0^{+}\right)=2.04 \pm 0.04(1.04 \pm 0.05) \mathrm{MeV}[14,15]$.

The $\Lambda$ separation energies in the $0^{+}$and $1^{+}$states of ${ }_{\Lambda}^{4} \mathrm{He}$, calculated using the Malfliet-TjonV-UCOM $V_{N N}(\mathrm{MTV})$ and YNG-NF $V_{\Lambda N}(\mathrm{NF})$ potentials are shown in Figure 1. The results of the variation of a basic FMD trial state $(\mathrm{V})$ are compared with the results with the even parity projected trial FMD state $\left(\mathrm{VAP}^{+}\right)$. Clearly, the $\mathrm{VAP}^{+}$leads to larger $\Lambda$ separation energies of the both $0^{+}$ and $1^{+}$states while the $0^{+}-1^{+}$splitting remains almost unchanged. This finding holds for all $V_{N N}$ and $V_{\Lambda N}$ potentials used in our calculations.

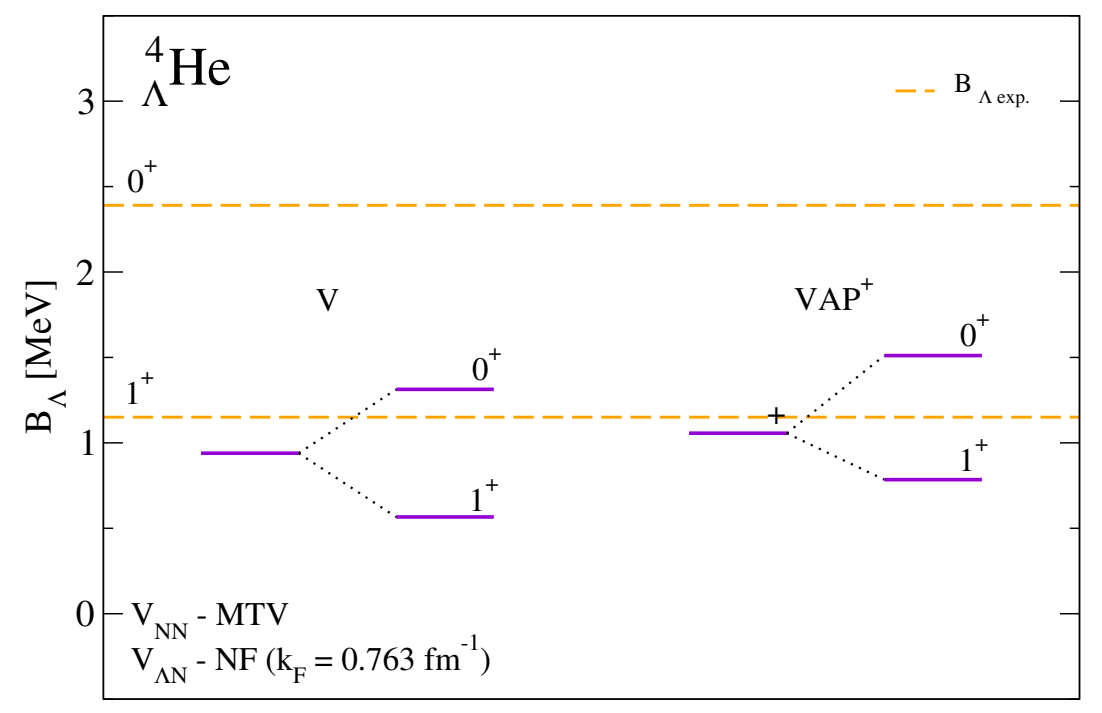

Figure 1: $\Lambda$ separation energies $B_{\Lambda}$ of ${ }_{\Lambda}^{4} \mathrm{He}$ obtained using the variation of a trial FMD state $(\mathrm{V})$ and variation of an even parity projected trial FMD state $\left(\mathrm{VAP}^{+}\right)$, calculated with the MTV $V_{N N}$ and NF $V_{\Lambda N}$ potentials. Variated trial states are further projected on the $0^{+}$ground and $1^{+}$excited states.

It is to be noted that the results of previous calculations of ordinary nuclei [13] suggest that the effect of $\mathrm{VAP}^{\pi}$ would be larger in more deformed hypernuclei. In the following we will present only the $\mathrm{VAP}^{\pi}$ results which we consider a more accurate description of hypernuclear bound states.

To a first approximation, the binding of the $\Lambda$ hyperon in a nuclear system is mainly determined by the $\Lambda N$ interaction. However, since the nuclear part of the total Hamiltonian (2.3) also participates in the variation process, the impact of the presence of the hyperon on the nuclear structure should depend on the $V_{N N}$ potential as well. This feature allows us to indicate the effects coming from the nuclear core description. In Figure 2, we plot the $\Lambda$ separation energies $B_{\Lambda}$ of the ${ }_{\Lambda}^{4} \mathrm{He}$ 


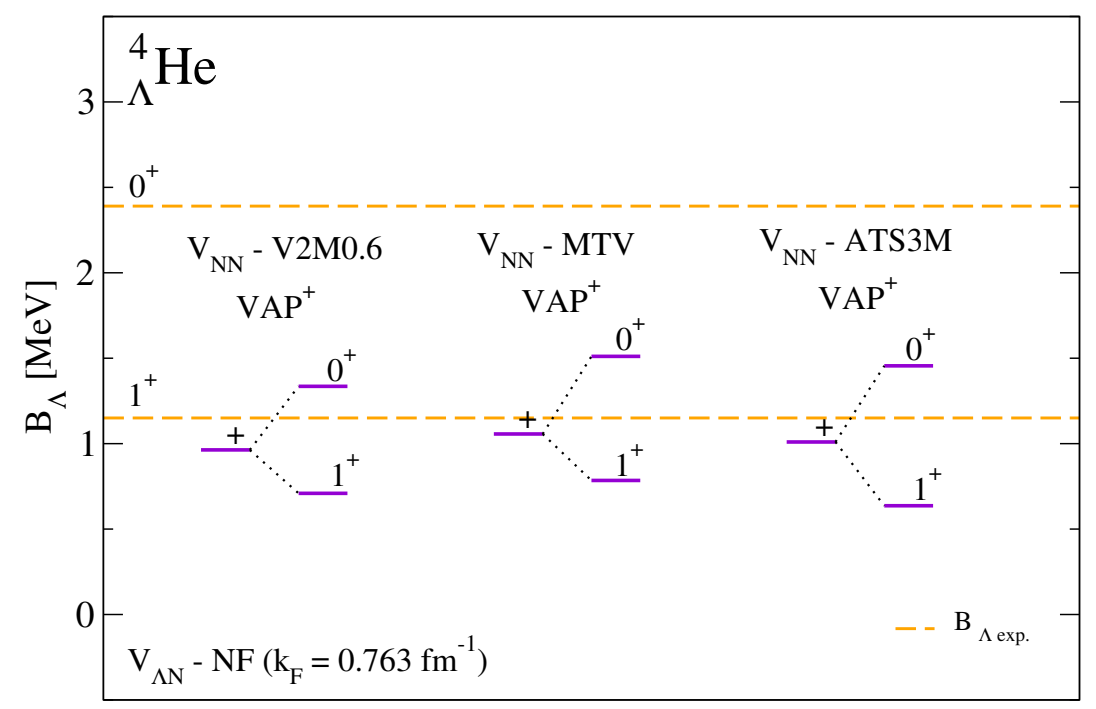

Figure 2: $\Lambda$ separation energies $B_{\Lambda}$ of ${ }_{\Lambda}^{4} \mathrm{He}$ obtained using the variation of an even parity projected trial FMD state $\left(\mathrm{VAP}^{+}\right)$calculated with the V2M0.6, MTV, and ATS3M $V_{N N}$ potentials and the NF $V_{\Lambda N}$ potential. Variated trial states $(+)$ are further projected on $0^{+}$ground and $1^{+}$excited states.

hypernucleus calculated for the same $V_{\Lambda N}$ potential (YNG-NF) but three different $V_{N N}$ potentials the MTV, Afnan-Tang-S3M-UCOM (ATS3M), and VolkovV2-M0.6 (V2M0.6) potentials. We observe, that the separation energy $B_{\Lambda}$ of an intrinsic state before the angular momentum projection (denoted by '+') varies only slightly within tens of keV. However, after the angular momentum projection the $\Lambda$ separation energies of the ground $0^{+}$and excited $1^{+}$states differ by few hundreds of $\mathrm{keV}$, which is also reflected in the $0^{+}-1^{+}$splitting.

In Table 1, we present energy differences between the individual contributions to the binding energies of the $0^{+}$and $1^{+}$states in ${ }_{\Lambda}^{4} \mathrm{He}$, calculated with the VolkovV2-M0.0 (V2M0.0), V2M0.6, MTV, and ATS3M $V_{N N}$ potentials and the NF $\left(k_{\mathrm{F}}=0.763 \mathrm{fm}^{-1}\right) V_{\Lambda N}$ potential. We observe that the size of the $\left|B_{\Lambda}\left(0^{+}\right)-B_{\Lambda}\left(1^{+}\right)\right|$splitting comes dominantly from the spin-dependent part of the $V_{\Lambda N}$ potential $\left(\Delta V_{\Lambda N}^{\sigma}\right)$. Furthermore, the size of $B_{\Lambda}$ splitting changes considerably for various $V_{N N}$ potentials, as they predict a different level of the nuclear core modifications due to the presence of $\Lambda$. Indeed, the ATS3M potential gives the largest change of the rms radius of ${ }^{3} \mathrm{He}$ and the nuclear

\begin{tabular}{lccccc|c}
$V_{N N}$ potential & $\Delta T_{\text {int. }}$ & $\Delta V_{N N}$ & $\Delta V_{N N}^{\sigma}$ & $\Delta V_{\Lambda N}$ & $\Delta V_{\Lambda N}^{\sigma}$ & $\left|B_{\Lambda}\left(0^{+}\right)-B_{\Lambda}\left(1^{+}\right)\right|$ \\
\hline V2M0.0 & $10^{-4}$ & $10^{-4}$ & - & $10^{-5}$ & 0.626 & 0.626 \\
V2M0.6 & $10^{-4}$ & $10^{-5}$ & $10^{-5}$ & $10^{-4}$ & 0.625 & 0.626 \\
MTV-UCOM & $10^{-4}$ & $10^{-5}$ & - & $10^{-4}$ & 0.726 & 0.726 \\
ATS3M-UCOM & 0.023 & 0.027 & 0.048 & 0.006 & 0.767 & 0.817
\end{tabular}

Table 1: The differencies between the $B_{\Lambda}\left(0^{+}\right)$and $B_{\Lambda}\left(1^{+}\right) \Lambda$ separation energies expressed in the form of individual contributions to the $0^{+}-1^{+}$splitting (in MeV). The $T_{i n t}, V_{N N}, V_{N N}^{\sigma}, V_{\Lambda N}$ and $V_{\Lambda N}^{\sigma}$ denote intrinsic kinetic energy, spin-independent part of $V_{N N}$, spin-dependent part of $V_{N N}$, spin-independent part of $V_{\Lambda N}$ and spin-dependent part of $V_{\Lambda N}$. The calculations are performed with the V2M0.0, V2M0.6, MTV, and ATS3M $V_{N N}$ potentials and the $\mathrm{NF}\left(k_{\mathrm{F}}=0.763 \mathrm{fm}^{-1}\right) V_{\Lambda N}$ potential. 
core of ${ }_{\Lambda}^{4} \mathrm{He}$.

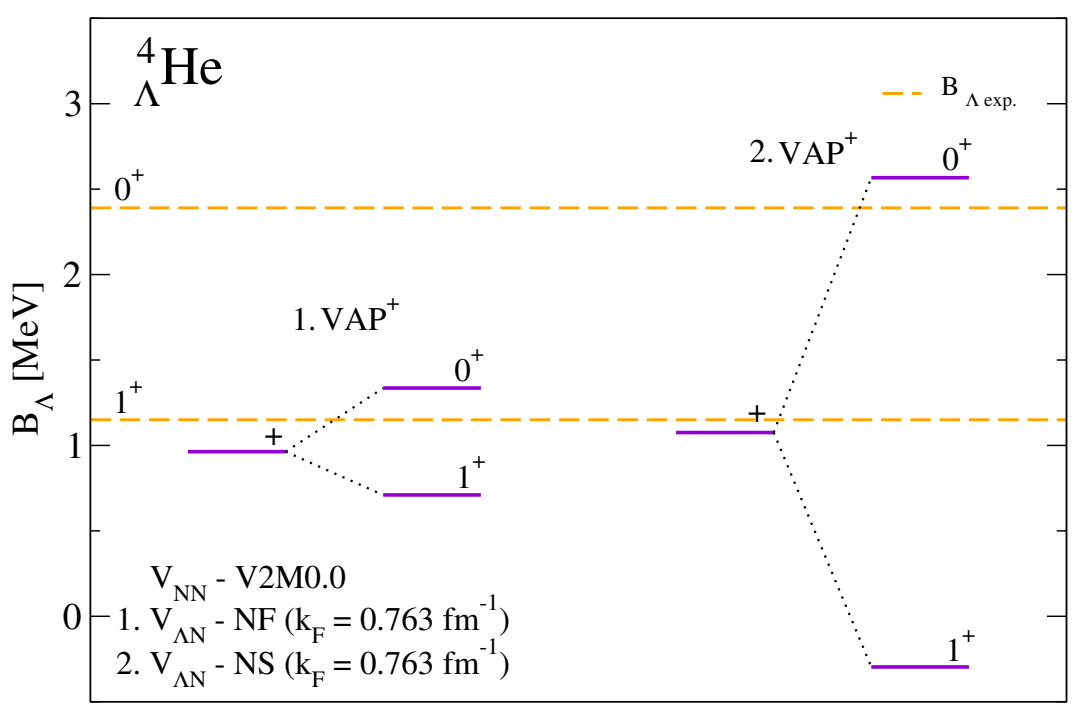

Figure 3: $\Lambda$ separation energies $B_{\Lambda}$ of ${ }_{\Lambda}^{4} \mathrm{He}$ obtained using the variation of an even parity projected trial FMD state $\left(\mathrm{VAP}^{+}\right)$calculated with the V2M0.0 $V_{N N}$ potential and NF, NS $V_{\Lambda N}$ potentials. Variated trial states $(+)$ are further projected on $0^{+}$ground and $1^{+}$excited states.

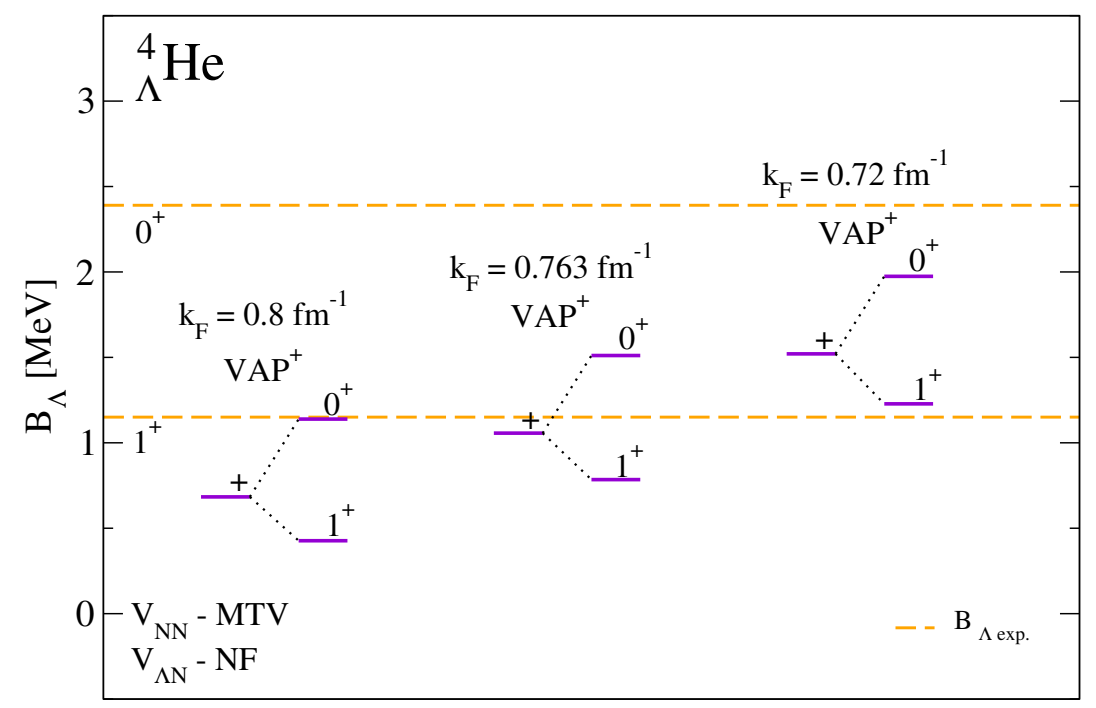

Figure 4: $\Lambda$ separation energies $B_{\Lambda}$ of ${ }_{\Lambda}^{4} \mathrm{He}$ obtained using the variation of an even parity projected trial FMD state $\left(\mathrm{VAP}^{+}\right)$calculated with the MTV $V_{N N}$ potential. The $V_{\Lambda N}$ part is described by the NF potential with different values of the Fermi momentum $k_{\mathrm{F}}$. Variated states $(+)$ are further projected on $0^{+}$ground and $1^{+}$excited states.

In Figure 3, we show the $\Lambda$ separation energies in ${ }_{\Lambda}^{4} \mathrm{He}$ calculated using two different $V_{\Lambda N}$ potentials, YNG-NF and YNG-NS, and the same V2M0.0 $V_{N N}$ potential. We observe substantial difference between the calculated $B_{\Lambda}$ energies as well as $0^{+}-1^{+}$splittings. The NS potential clearly overestimates the $\left|B_{\Lambda}\left(0^{+}\right)-B_{\Lambda}\left(1^{+}\right)\right|$difference which is attributed to the strong spin dependent part of the $\Lambda N$ interaction. This feature is in accordance with previous calculations [4]. 
The important aspect of the YNG potentials is their Fermi momentum dependence. The calculations presented so far were performed for one particular value of the Fermi momentum, $k_{\mathrm{F}}=0.763 \mathrm{fm}^{-1}$, determined from the calculations of the ${ }^{3} \mathrm{He}$ rms radius [16]. In Figure 4, we plot the results of three different calculations of ${ }_{\Lambda}^{4} \mathrm{He}$ with the same $V_{N N}$ and $V_{\Lambda N}$ potentials, but different values of $k_{\mathrm{F}}$. The value $k_{\mathrm{F}}=0.8 \mathrm{fm}^{-1}$ was adopted from previous hypernuclear cluster calculations [4] and $k_{F}=0.72 \mathrm{fm}^{-1}$ was used as a test value. We observe that the Fermi momentum acts as a scaling factor $-\Lambda$ separation energies increase rapidly with decreasing $k_{\mathrm{F}}$. The $0^{+}-1^{+}$splitting changes only slightly (tens of $\mathrm{keV}$ ) and also has an increasing tendency with decreasing $k_{\mathrm{F}}$.

Properties of the $N N$ and $\Lambda N$ interactions can be studied through mirror hypernuclei ${ }_{\Lambda}^{4} \mathrm{He}$ and ${ }_{\Lambda}^{4} \mathrm{H}$. Since the YNG potentials do not distinguish between neutron and proton, the difference between the $\Lambda$ separation energies comes exclusively from the $N N$-potential description of the nuclear core. In Figure 5, we plot the $B_{\Lambda}$ energies in the aforementioned systems. The calculation was performed with the ATS3M $V_{N N}$ potential which distinguishes between individual isospin channels. As a result, we received a slightly higher-lying spectrum for ${ }_{\Lambda}^{4} \mathrm{H}$ (the difference is about tens of $\mathrm{keV})$.

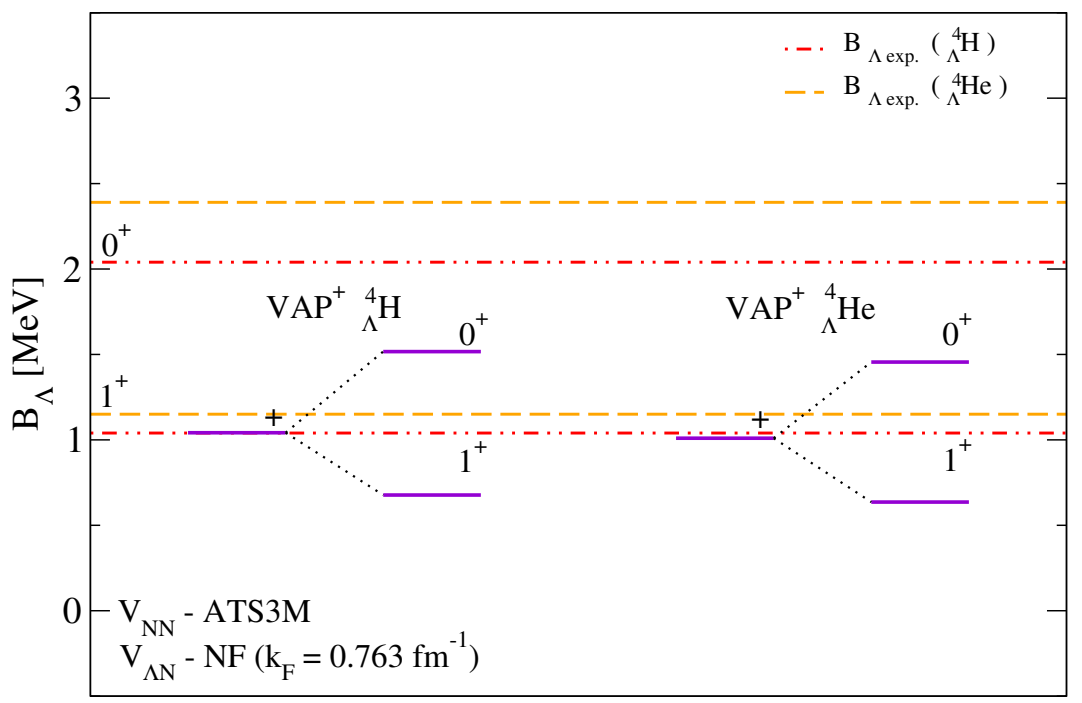

Figure 5: $\Lambda$ separation energies $B_{\Lambda}$ of ${ }_{\Lambda}^{4} \mathrm{He}$ and ${ }_{\Lambda}^{4} \mathrm{H}$ obtained using the variation of an even parity projected trial FMD state $\left(\mathrm{VAP}^{+}\right)$. Calculations are performed with the ATS3M $V_{N N}$ potential and NF $V_{\Lambda N}$ potential. Variated states $(+)$ are further projected on $0^{+}$ground and $1^{+}$excited states.

\section{Summary}

We performed first calculations of s-shell $\Lambda$ hypernuclei using a recently developed FMD code. In this contribution, we presented selected results for ${ }_{\Lambda}^{4} \mathrm{He}$ and ${ }_{\Lambda}^{4} \mathrm{H}$ in order to discuss main features of the calculations. Applying the parity projection techniques to the trial FMD states before variation yields larger separation energies of the $\Lambda$ hyperon. We found rather weak dependence of the calculated $\Lambda$ separation energies on the applied $V_{N N}$ potential. On the other hand, the proper choice of the $V_{\Lambda N}$ potential, as well as $k_{\mathrm{F}}$ which enters the YNG potentials, appeared crucial for the predictions of the $\Lambda$ separation energies and $0^{+}-1^{+}$splitting. 
As a next step, we should incorporate more sophisticated $V_{N N}$ and $V_{\Lambda N}$ potentials, such as those derived from chiral effective field theory. Moreover, the $\Lambda-\Sigma$ mixing which plays important role in realistic hypernuclear calculations should be included. It is also desirable to study the role of three body forces. We intend to extend our calculations to p-shell hypernuclei and investigate the effect of the $\Lambda$ hyperon on the intrinsic nuclear clasterization.

\section{Acknowledgments}

The speaker thanks the organizers for the student fellowship support. This work was supported by GACR Grants No. P203/12/2126 and No. 04301S. M.S. acknowledges financial support of the Grant Agency of the Czech Technical University in Prague, grant No. SGS13/215/OHK4/3T/14. We would like to thank J. Hrtankova for careful reading of the manuscript.

\section{References}

[1] P. Bydzovsky, A. Gal, J. Mares (Eds.), Topics in Strangeness Nuclear Physics (Springer, 2007)

[2] H. Feldmeier, Nucl. Phys. A 515 (1990) 147

[3] H. Feldmeier, K.Bieler, J. Schnack., Nucl. Phys. A 586 (1995) 493

[4] Y. Kanada-En'Yo, H. Horiuchi, PTP. Suppl. 142 (2001)

[5] M. Isaka, M. Kimura, A. Dote, A. Ohnishi, Phys. Rev. C 83 (2011) 044323

[6] A. Volkov, Nucl. Phys. 74 (1965) 33

[7] R. A. Malfliet, J. A. Tjon, Nucl. Phys. A 127 (1969) 161

[8] I. R. Afnan, Y. C. Tang, Phys. Rev. 175 (1968) 1337

[9] H. Feldmeier, T. Neff, R. Roth, J. Schnack, Nucl. Phys. A 632 (1998) 61

[10] Y. Yamamoto et al., PTP Suppl. 117 (1994) 361

[11] P. Spellucci, Math. Prog. 82 (1998) 413

[12] T. Ring, P. Schuck, The Nuclear Many-Body Problem (Springer, 2000)

[13] T. Neff, H. Feldmeier, Eur. Phys. J. Special Topics 156 (2008) 69

[14] D. H. Davis, J. Pniewski, Contemp. Phys. 27 (1986) 91

[15] M. Bedjidian et al., Phys. Lett. B 83 (1979) 252

[16] M. Isaka, private communication 